\title{
Mitotic spindle destabilization and genomic instability in Shwachman-Diamond syndrome
}

\author{
Karyn M. Austin, ${ }^{1}$ Mohan L. Gupta Jr., ${ }^{2}$ Scott A. Coats, ${ }^{3}$ Asmin Tulpule, ${ }^{1}$ Gustavo Mostoslavsky, ${ }^{4}$ \\ Alejandro B. Balazs, ${ }^{4}$ Richard C. Mulligan, ${ }^{4}$ George Daley,,${ }^{1,2,5}$ \\ David Pellman, ${ }^{1,2,5}$ and Akiko Shimamura ${ }^{1,2,3,5}$

\begin{abstract}
'Department of Pediatric Hematology, Children's Hospital Boston, Boston, Massachusetts, USA. 2Department of Pediatric Oncology, Dana Farber Cancer Institute, Boston, Massachusetts, USA. ${ }^{3}$ Department of Pediatric Hematology/Oncology, University of Washington/

Children's Hospital and Regional Medical Center, Seattle, Washington, USA. ${ }^{4}$ Department of Genetics, Harvard Medical School, and Division of Molecular Medicine, Children's Hospital Boston, Boston, Massachusetts, USA. ${ }^{5}$ Harvard Medical School, Boston, Massachusetts, USA.
\end{abstract}

\begin{abstract}
Deficiencies in the SBDS gene result in Shwachman-Diamond syndrome (SDS), an inherited bone marrow failure syndrome associated with leukemia predisposition. SBDS encodes a highly conserved protein previously implicated in ribosome biogenesis. Using human primary bone marrow stromal cells (BMSCs), lymphoblasts, and skin fibroblasts, we show that SBDS stabilized the mitotic spindle to prevent genomic instability. SBDS colocalized with the mitotic spindle in control primary BMSCs, lymphoblasts, and skin fibroblasts and bound to purified microtubules. Recombinant SBDS protein stabilized microtubules in vitro. We observed that primary BMSCs and lymphoblasts from SDS patients exhibited an increased incidence of abnormal mitoses. Similarly, depletion of SBDS by siRNA in human skin fibroblasts resulted in increased mitotic abnormalities and aneuploidy that accumulated over time. Treatment of primary BMSCs and lymphoblasts from SDS patients with nocodazole, a microtubule destabilizing agent, led to increased mitotic arrest and apoptosis, consistent with spindle destabilization. Conversely, SDS patient cells were resistant to taxol, a microtubule stabilizing agent. These findings suggest that spindle instability in SDS contributes to bone marrow failure and leukemogenesis.
\end{abstract}

\section{Introduction}

Shwachman-Diamond syndrome (SDS) is an autosomal recessively inherited disorder characterized by bone marrow failure, exocrine pancreatic insufficiency, and an increased risk of acute myeloid leukemia (reviewed in refs. 1,2). Patients with SDS exhibit abnormalities in a variety of additional organs, including bone, liver, and immunologic and endocrine systems. SDS is associated with mutations in the SBDS gene (3), which encodes a highly conserved novel protein with a broad tissue expression pattern. Targeted disruption of Sbds leads to early embryonic lethality in mice (4). SBDS protein expression is markedly reduced but not absent in most SDS patients harboring biallelic SBDS mutations (refs. 5, 6, and Supplemental Figure 1; supplemental material available online with this article; doi:10.1172/JCI33764DS1). To date, no patient harboring 2 null SBDS alleles has been identified, suggesting that complete absence of the SBDS protein is lethal in humans as it is in mouse models. Knockdown of SBDS protein levels with siRNA in murine hematopoietic cells results in impaired myeloid cell production (7).

Patients with SDS have a propensity to develop clonal cytogenetic abnormalities in the bone marrow $(8,9)$, and leukemias arising in these patients commonly exhibit aneuploidy and complex cytogenetic abnormalities $(1,2)$. Whether genomic instability reflects a direct effect of SBDS loss or rather represents a second-

Nonstandard abbreviations used: BMSC, bone marrow stromal cell; SDS, Shwachman-Diamond syndrome.

Conflict of interest: The authors have declared that no conflict of interest exists. Citation for this article: J. Clin. Invest. 118:1511-1518 (2008). doi:10.1172/JCI33764. ary event is not known. Here we examine the effects of SBDS loss during cell division and identify a previously unanticipated role for SBDS in mitosis.

\section{Results}

Mitotic aberrations are increased in SDS patient cells and following SBDS knockdown. SBDS genetic mutations and SBDS protein expression in our SDS patient cohort has been previously reported (6) (see Supplemental Table 1). The SBDS mutations IVS2+2T $\rightarrow \mathrm{C}$ and c183_184 TA $\rightarrow$ CT are the most common mutations in SDS patients, as reflected in our patient cohort (3). Here we examined the morphology of mitotic primary bone marrow stromal cells (BMSCs) derived from patients with SDS (Figure 1A). Analysis of BMSCs of similar passage from normal controls versus SDS patients revealed an increased percentage of abnormal metaphases with centrosomal amplification and multipolar spindles in the patient-derived BMSCs (Figure 1A). This increase in mitotic abnormalities was observed in BMSCs derived from 3 different SDS patients. Centrosomal amplification was also noted in lymphoblast cell lines from SDS patients as compared with healthy normal controls (Supplemental Figure 2).

To determine whether mitotic abnormalities were the result of $S B D S$ loss, we infected an immortalized skin fibroblast cell line (GM00038) with lentiviral vectors containing siRNA against either SBDS or against luciferase. Introduction of SBDS siRNA resulted in a marked reduction of SBDS protein levels by day 3 after infection, as ascertained by western blotting (Figure 1B). Early in the course of SBDS protein knockdown, few cells with multipolar spindles and centrosomal amplification were noted; however, 

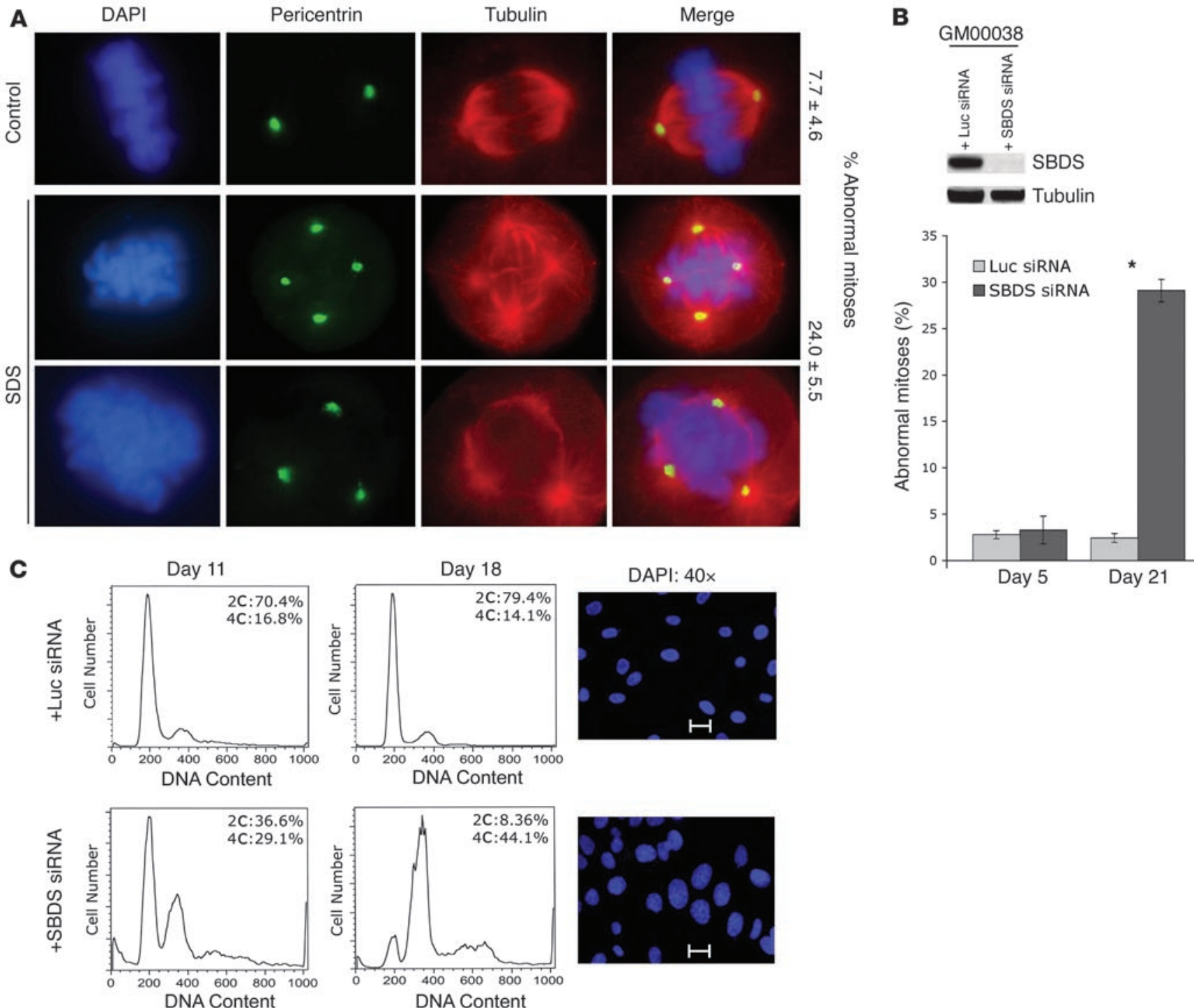

\section{Figure 1}

SBDS loss promotes mitotic abnormalities. (A) SBDS ${ }^{-1-}$ cells exhibit mitotic abnormalities. Primary BMSCs from at least 3 different SDS patients were fixed and stained with antibodies against pericentrin (green) and $\alpha$-tubulin (red) and with DAPI (blue). The top row illustrates normal control metaphase staining, while the middle and bottom rows illustrate the aberrant mitotic figures observed in SDS patient cells. Note multiple centrosomes, multipolar spindles, and broad DNA distribution. The percentage of abnormal mitotic cells in controls versus $S B D S^{-/-}$cells is noted on the right $(P<0.01)$. A minimum of 200 cells were counted per sample in a blinded fashion in 3 independent experiments. Original magnification, $\times 60$. (B) Targeted SBDS loss results in aberrant mitosis. GM00038 or GM00637 skin fibroblast cell lines immortalized with SV40 T antigen were infected with dual expression cassette lentiviral constructs encoding both GFP and siRNA sequences, the latter targeted against either SBDS or a LUC control. GFP-positive cells were sorted and analyzed for SBDS expression by western blot. SBDS protein expression was markedly reduced by 3 days following infection (upper panel). On day 5 and day 21 following infection, the cells were fixed and stained with antibodies against pericentrin and tubulin. At least 150 cells per sample were scored for abnormal mitoses (centrosomal amplification and multipolar spindles as illustrated in A) in a blinded fashion in at least 3 independent experiments, and the percentage of abnormal mitoses were tabulated in the histogram. ${ }^{*} P=0.01$, LUC siRNA compared with SBDS siRNA on day 21. (C) SBDS loss results in aneuploidy. GM00038 cells from B were infected with lentivirus vectors as described in B. These immortalized GM00038 cells failed to exhibit p53-dependent p21 upregulation following exposure to ionizing radiation. GFP-positive cells were gated and analyzed for DNA content by flow cytometry on the indicated days following infection. DAPI staining shows enlargement of nuclei for cells lacking SBDS. Cells were visualized under $\times 40$ magnification, and a scale bar (arbitrary units) is shown in $\mathbf{C}$ for comparison of the top and bottom panels.

such mitotic abnormalities became increasingly prominent over the ensuing 3 weeks of culture in the presence of SBDS siRNA but not with the control siRNA (Figure 1B). Two independent immortalized skin fibroblast cell lines yielded similar results following
SBDS siRNA introduction. We attempted to complement the $S B D S^{-/-}$patient-derived cells by retroviral-mediated delivery of the wild-type SBDS cDNA. Although we were able to introduce SBDS in short-term culture (2-3 days; see below), chronic overexpression 
of SBDS protein in primary BMSCs or lymphoblasts resulted in rare mitotic cells and poor cell growth such that we were unable to derive cell lines stably overexpressing wild-type SBDS.

We next investigated the effects of SBDS loss on chromosomal content. Comparison of DNA content analyzed by flow cytometry for control primary BMSCs versus $S B D S^{-/-}$patient-derived BMSCs showed a sub-G $\mathrm{G}_{1}$ population of $S B D S^{-/-}$cells (Supplemental Figure 3 ), which was confirmed to represent an increased apoptotic population as assayed by PARP cleavage. Introduction of SBDS siRNA into wild-type primary BMSCs was accompanied by increased cell death and poor growth (K. Ganapathi and A. Shimamura, unpublished observations). We reasoned that this might represent apoptotic elimination of abnormal mitotic cells through activation of cellular checkpoints to prevent the propagation of chromosomal abnormalities. Indeed, p53 protein levels and p21 expression increased in response to ionizing radiation in primary BMSCs from SDS patients, indicating that p53 checkpoint responses were intact (Supplemental Figure 4). We therefore examined the effect of knocking down SBDS protein levels in each of 2 human skin fibroblast cell lines (GM00038 and GM00637), wherein the p53 checkpoint had been inactivated by SV40 large T antigen. By 11-18 days following SBDS knockdown, a broad population of cells containing greater than 4C DNA content was increasingly observed in the cells infected with the SBDS siRNA lentivirus (Figure 1C). By fluorescence microscopy, a population of cells with enlarged nuclei consistent with increased DNA content was observed in the cells lacking SBDS (Figure 1C). In contrast, the flow cytometry profile of the fibroblasts infected with the control luciferase siRNA lentivirus remained stable. Thus, we concluded that loss of SDS induces polyploidy/aneuploidy together with a progressive accumulation of cells undergoing multipolar mitoses.

$S B D S$ colocalizes with the mitotic spindle. To investigate the potential role of SBDS in mitosis, we examined SBDS intracellular localization in metaphase cells from primary BMSCs from healthy controls. A fraction of the SBDS signal was concentrated on the mitotic spindle, most obviously near the spindle poles, as confirmed by costaining for tubulin (Figure 2A). Three lines of evidence support the specificity of the spindle-associated SBDS signal. First, no spindle staining was observed in $S B D S^{-/-}$patient-derived BMSCs, which largely lacked SBDS protein expression (Figure 2A). Second, shortterm overexpression of the wild-type SBDS cDNA in these cells (Figure $2 \mathrm{~B}$ ) restored the spindle labeling (Figure $2 \mathrm{~B}$ ), though mitotic cells were rare following SBDS overexpression. Finally, the SBDS spindle labeling was abolished after SBDS knockdown (Figure 2C).

We also assessed the intracellular localization of SBDS in interphase cells costained for tubulin (Figure 2A). SBDS was found in both the nucleus and cytoplasm; however, SBDS exhibited a diffuse granular cytoplasmic pattern distinct from the linear pattern of localization seen with tubulin. Although SBDS does not obviously colocalize with tubulin in interphase cells, we cannot exclude an association of a small proportion of SBDS protein with interphase microtubules.

$S B D S$ binds and stabilizes microtubules in vitro. To determine whether SBDS binds microtubules in vitro, purified taxol-stabilized microtubules were added to cell lysates containing endogenous SBDS protein. The microtubules were separated from unbound proteins by centrifugation through a glycerol cushion. The presence of endogenous SBDS protein associated with the microtubule pellet was determined by western blot. Without the addition of stabilized microtubules, no SBDS was found in the pellet (Figure 3A, lane 1).
When increasing concentrations of microtubules were added to the cell lysate, SBDS protein proportionally co-pelleted with the microtubules (Figure 3A, lanes 2-5).

The binding of SBDS to microtubules can occur independently of other microtubule-binding proteins because purified, recombinant SBDS produced in bacteria (which lacks microtubule binding proteins) still bound microtubules with an estimated $K_{d}$ of $7.6 \pm 3.0$ $\mu \mathrm{M}$ (Figure 3B). Additionally, intracellular spindle binding may be stabilized by additional proteins or posttranslational modifications (10). Based on western blotting against a standard curve of purified SBDS or tubulin, we estimated that the molar ratio of SBDS to tubulin was around 1:50. Since the concentration of tubulin in HeLa cells has been estimated at $10 \mu \mathrm{M}$, by these calculations the cellular concentration of SBDS was approximately $0.2 \mu \mathrm{M}$.

Using purified components, we determined that recombinant SBDS protein stabilized microtubules in vitro. Upon dilution of preformed microtubules, the spontaneous depolymerization into free tubulin dimers was monitored by a fluorescence assay. (Figure 3C, PBS control). When increasing concentrations of purified SBDS protein were added to the reaction, a dose-dependent protection against dilution-induced depolymerization was noted (Figure 3C). As a positive control, the addition of taxol, a known microtubule stabilizer, similarly promoted microtubule stabilization in this assay. As a negative control, the addition of bovine serum albumen protein did not promote microtubule stabilization (Supplemental Figure 5). Together, these data indicate that SBDS protein directly binds and stabilizes microtubules in vitro.

SDS patient cells are sensitive to nocodazole and resistant to taxol. To assess the effects of microtubule destabilization in SDS patient cells, we examined the effect of the microtubule-destabilizing drug, nocodazole, on lymphoblast cell lines from $S B D S^{-/-}$patients. $S B D S^{-/}$patient cell lines exhibited a rapid drop in cell viability at concentrations of nocodazole that depolymerize microtubules, whereas viability of the normal control was not affected until significantly higher concentrations (Figure 4A). Following nocodazole treatment, SDS patient cells also exhibited increased apoptosis, as measured by PARP cleavage (Supplemental Figure $6 \mathrm{~A}$ ), and annexin $\mathrm{V}$ binding (Supplemental Figure 6C), compared with normal controls. Short-term introduction of a wild-type SBDS cDNA into $S B D S^{-/-}$patient cells partially corrected this hypersensitivity to nocodazole; thus, the nocodazole sensitivity of patient cells was SBDS dependent (Figure 4C).

We next investigated the effects of nocodazole on mitosis in $S B D S^{-/-}$cells. Abnormalities of the mitotic spindle trigger the mitotic spindle checkpoint to prevent subsequent aberrant chromosomal segregation. To investigate whether the mitotic abnormalities observed in the SDS patient cells might result from loss of the mitotic spindle checkpoint, we treated healthy control cells and SDS patient cells with low doses of nocodazole. The nocodazole-induced mitotic spindle instability should trigger a mitotic delay if the mitotic spindle checkpoint is active (11). As shown in Figure 4E, a dose-dependent increase in the mitotic index was observed following nocodazole treatment of BMSCs from healthy controls or SDS patients. Thus, the mitotic spindle checkpoint is intact in $S B D S^{-/}$cells. Indeed, cells deficient in SBDS exhibited a higher mitotic index (percentage of cells in metaphase) in a dose-dependent manner than did normal control cells (Figure 4E). To further investigate whether the increased activation of the mitotic checkpoint was dependent on SBDS, we also knocked down SBDS expression with siRNA in immortalized skin fibro- 
A
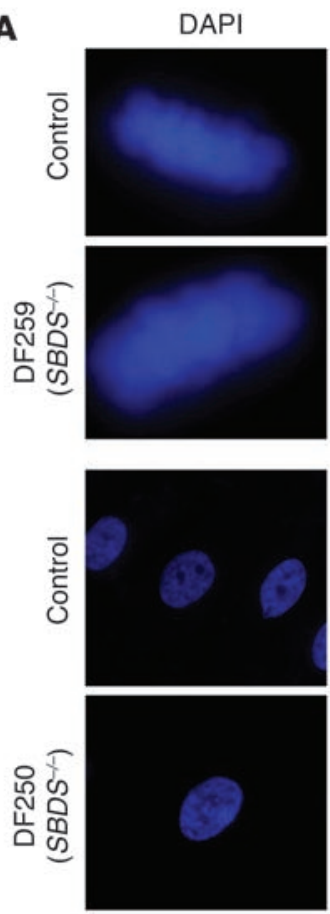

B

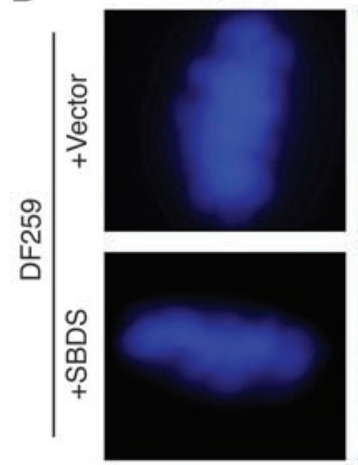

C

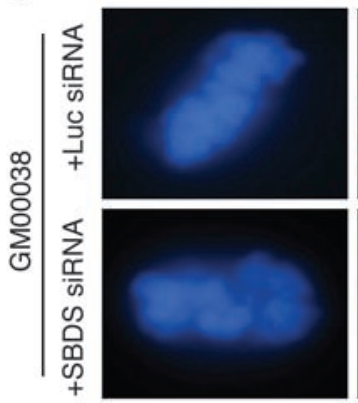

SBDS
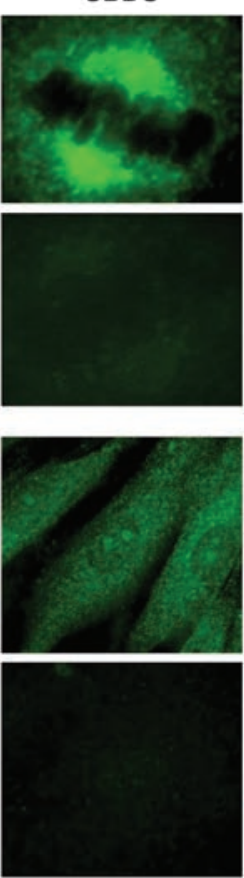

SBDS
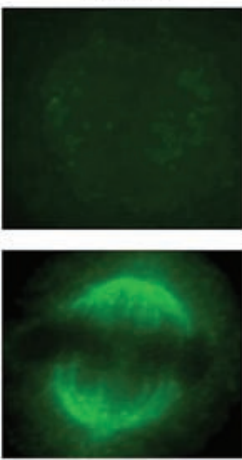

SBDS
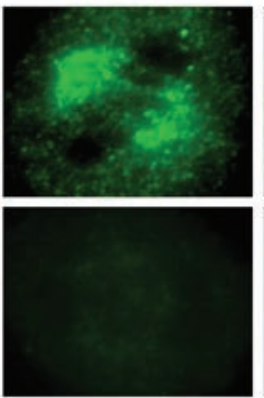

Tubulin
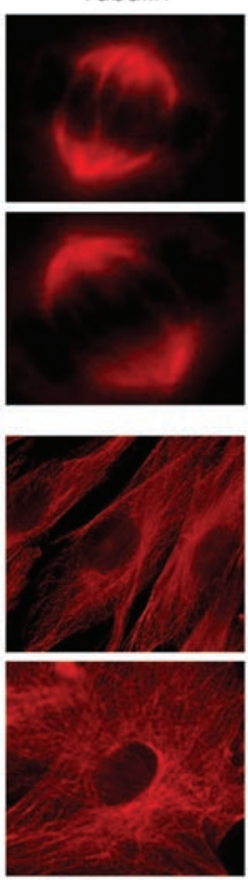

Tubulin
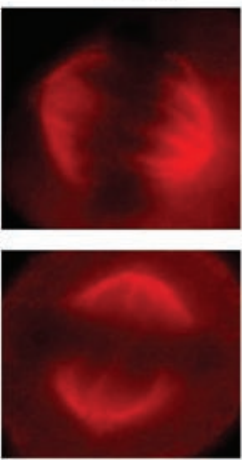

Merge
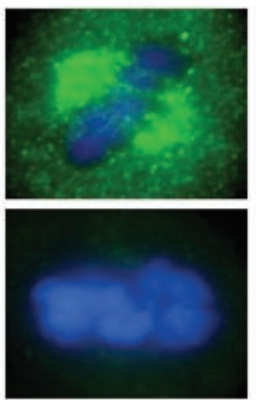
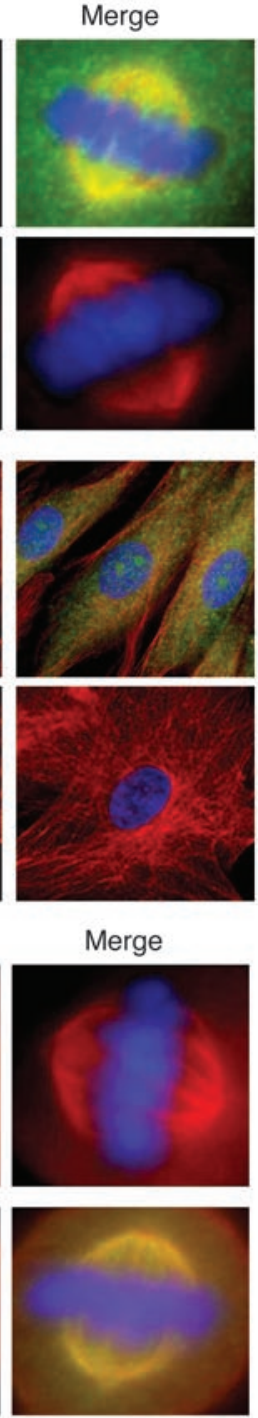

SBDS

Tubulin

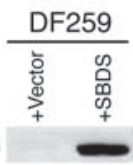

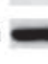

\section{Figure 2}

SBDS localizes to a region corresponding to that of the mitotic spindle. (A) SBDS colocalizes with the spindle by immunofluorescence. Primary BMSCs in metaphase (upper 2 panels) or in interphase (lower 2 panels) from normal control or $S B D S^{-/-}$patients were fixed and stained with antibodies against SBDS (green), $\alpha$-tubulin (red), and DAPI (blue). SBDS spindle staining was not detectable in any of the 6 SDS patients exhibiting low SBDS protein expression as analyzed by western blot. (B) The spindle-staining pattern is SBDS dependent. DF259 primary BMSCs were infected with a retroviral vector carrying SBDS cDNA or an empty vector control, as indicated. Cells were lysed or fixed for immunofluorescence studies 48-72 $\mathrm{h}$ after infection. Western blot analysis for SBDS expression is shown on the right. Tubulin was stained to control for equal sample loading. Fixed cells were stained for SBDS (green), $\alpha$-tubulin (red), or DAPI (blue) and visualized by fluorescence microscopy. (C) Loss of SBDS abrogates the spindle-staining pattern. GM00038 skin fibroblasts were infected with lentiviral siRNA vectors against either SBDS or a LUC control and analyzed by immunofluorescence for SBDS (green). 
A

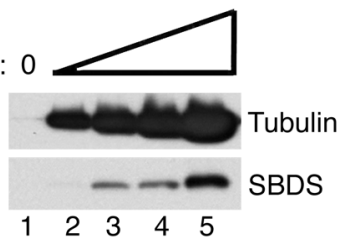

B

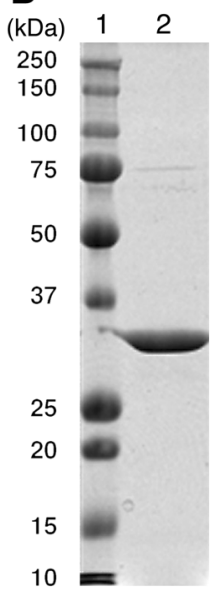

Tubulin $(\mu \mathrm{M})$

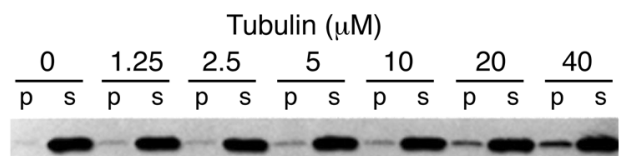

C

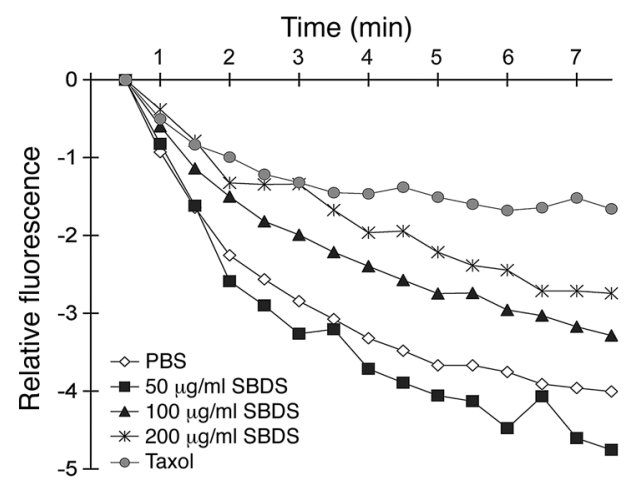

blasts and observed the same increase of the nocodazole-induced mitotic index noted in SDS patient cells (Supplemental Figure 7). Thus, loss of SBDS synergizes with the spindle-destabilizing effects of nocodazole to trigger cell cycle arrest, consistent with spindle destabilization in the absence of SBDS.

We next examined the effect of taxol, a microtubule-stabilizing drug, on $\mathrm{SBDS}^{-/-}$cell viability. Taxol disrupts normal microtubule dynamics by promoting microtubule polymerization and inhibiting microtubule disassembly (12). $S B D S^{-/-}$patient lymphoblasts were resistant to taxol-induced cell death and apoptosis relative to controls (Figure 4B and Supplemental Figure 6, B and D). Shortterm introduction of a wild-type $S B D S$ cDNA into $S B D S^{-/-}$patient cells partially restored taxol sensitivity (Figure 4D).

We examined the effects of taxol on mitosis in $S B D S^{-/-}$cells. Primary BMSCs from normal controls or $S B D S^{-/-}$patients were exposed to increasing doses of taxol, and the percentage of cells in metaphase was determined. In general, primary BMSCs did not exhibit a robust cell cycle arrest in response to taxol; however, $S B D S^{-/-}$cells were relatively resistant to mitotic delay following taxol treatment as compared with controls (Figure 4F). Mitotic delay could not be assessed at doses of taxol above $10 \mathrm{nM}$, due to extensive cell death. Interestingly, we also observed that treat-

\section{Figure 3}

SBDS binds to and stabilizes microtubules in vitro. (A) Endogenous SBDS co-pellets with microtubules from cell lysates. Increasing concentrations of purified, taxol-stabilized bovine microtubules were incubated with HeLa cell extract, layered over a glycerol cushion, and centrifuged to pellet the microtubules and associated proteins. The pellets were analyzed by western blot for tubulin and SBDS. (B) Recombinant SBDS co-sediments with microtubules. Left: Coomassie-stained gel loaded with standard molecular markers (lane 1) or $3 \mu \mathrm{g}$ purified recombinant SBDS protein (lane 2). Upper right: The fraction of 500 $\mathrm{nM}$ SBDS remaining in the supernatant $(s)$ or co-pelleting $(p)$ with increasing amounts of taxol-stabilized microtubules after centrifugation through a glycerol cushion was monitored by SBDS immunoblotting. Lower right: The average percentage bound from 3 experiments. The 1-site binding isotherm and $\mathrm{K}_{\mathrm{d}}=7.6 \pm 3.0 \mu \mathrm{M}$ were obtained by best-fit nonlinear regression using Graphpad Prism software. Approximately $14 \%$ of purified SBDS was co-pelleted with microtubules. Error bars represent SEM. (C) SBDS stabilizes microtubules in vitro. Preformed fluorescence-associated microtubules were diluted into PBS buffer alone or into buffer containing either taxol or increasing concentrations of purified, bacterially expressed recombinant SBDS protein. SBDS concentrations of $1.74 \mu \mathrm{M}, 3.5 \mu \mathrm{M}$, and $7 \mu \mathrm{M}$ were assayed. Dilution-induced depolymerization was monitored by the loss of microtubule-stimulated fluorescence signal. Three independent experiments yielded similar results. A representative experiment is shown. As a negative control, purified bovine serum albumin protein had no effect on microtubule stability (Supplemental Figure 4).

ment with 5-10 nM taxol resulted in the formation of micronuclei, which may be a consequence of lagging chromosomes or acentric DNA fragments. The presence of kinetochores within the micronuclei as determined by immunofluorescence staining indicated that these micronuclei likely result from lagging chromosomes rather than DNA fragments. Interestingly, $S B D S^{-/-}$cells exhibited a lower percentage of cells with taxol-induced micronuclei as compared with controls (Figure 4G); thus, SDS cells were relatively resistant to taxol. The localization of SBDS to mitotic spindles, its binding and stabilization of microtubules in vitro, and the reciprocal effects of SBDS loss on nocodazole and taxol sensitivity all support the hypothesis that SBDS stabilizes spindle microtubules.

\section{Discussion}

Our study demonstrates that SBDS promotes spindle stability and chromosome segregation. The spindle stability defect in SBDS cells may explain the high frequency of chromosomal abnormalities, commonly monosomy 7, observed in the bone marrows of SDS patients. Another human disease characterized by a mitotic defect and cancer susceptibility is the mosaic variegated aneuploidy syndrome (13) resulting from BUB1B mutations that are associated with premature chromatid separation and disruption of the spindle checkpoint. To our knowledge, SBDS provides the first reported example of a defect in a microtubule binding protein associated with cancer predisposition and marrow failure.

It is important to point out that SBDS deficiency does not result in immediate global mitotic disruption, but rather spindle abnormalities whose consequences appear to be cumulative over time. Our observations that multipolar spindles and centrosomal amplification were not observed immediately after SBDS knockdown but grew increasingly prominent over time raise the likely possibility that these abnormalities result from additional secondary events following spindle destabilization by SBDS loss. Previous studies have shown that cells with spindle abnormalities 

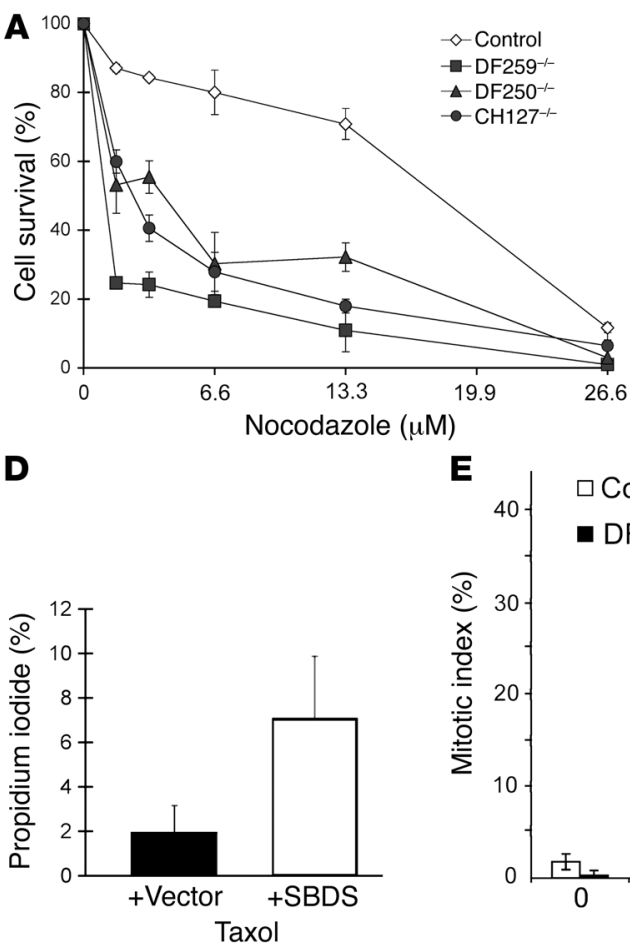

E
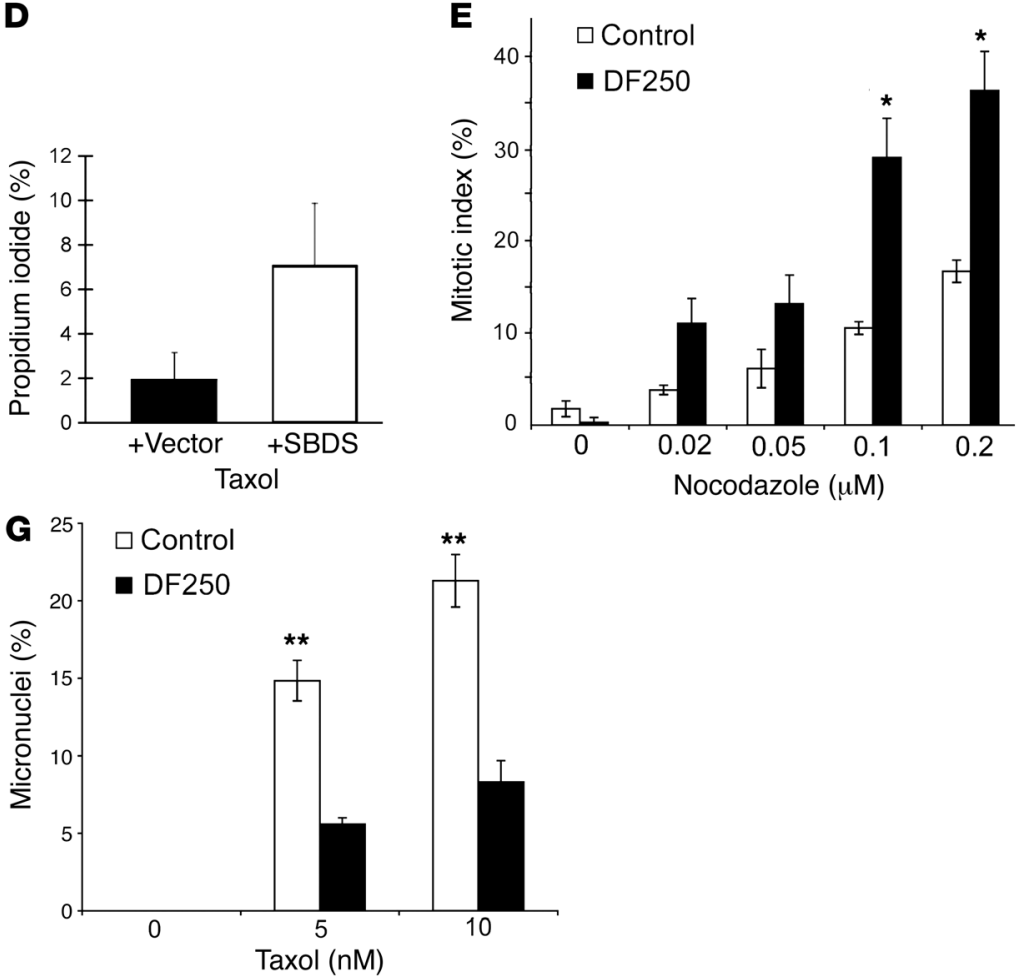

Figure 4

SBDS stabilizes microtubules in vivo. (A and B) SDS patient cells are sensitive to nocodazole and resistant to taxol. Lymphoblast cell lines originating from SDS patients (DF259, DF250, CH127, and DF1227) or normal control lymphoblasts were treated with increasing concentrations of nocodazole for $24 \mathrm{~h} \mathrm{(A)} \mathrm{or} \mathrm{taxol} \mathrm{for} 72 \mathrm{~h} \mathrm{(B).} \mathrm{Cell} \mathrm{survival} \mathrm{was} \mathrm{determined} \mathrm{using} \mathrm{AqueousOne} \mathrm{colorimetric} \mathrm{assay} \mathrm{reagent.} \mathrm{Each} \mathrm{cell} \mathrm{survival}$ assay was performed in triplicate for each experiment for a total of 3 independent experiments. SEM is denoted with vertical bars. (C and $\mathbf{D}$ ) Nocodazole sensitivity and taxol resistance are SBDS dependent. DF259 lymphoblasts were transiently infected with a dual expression lentiviral vector containing either GFP and SBDS cDNA or GFP alone (vector). Two days following infection, cells were treated with $6.6 \mu \mathrm{M}$ nocodazole (C) or $50 \mathrm{nM}$ taxol (D) for $18 \mathrm{~h}$. Cell death was assayed by flow cytometry for propidium iodide uptake. Three independent experiments were performed for each assay. $P<0.25$ (C) and $P<0.05$ (D). (E) $S B D S^{-/-}$cells exhibit an increased mitotic arrest with nocodazole. Normal control or SDS patient primary BMSCs were treated with increasing doses of nocodazole for $18 \mathrm{~h}$. Cells were stained with DAPI, and the percentage of cells in metaphase (expressed as the mitotic index) was visually scored for at least 100 cells in triplicate for 3 independent experiments. Samples were quantitated in a blinded fashion. The mitotic index was calculated from the total number of mitotic cells divided by the total cell number counted. BMSCs from 2 different SDS patients (DF250 and DF259) yielded similar results. ${ }^{*} P<0.01$. (F) $S B D S^{-l-}$ cells are resistant to taxol-induced mitotic arrest. Normal control or SBDS ${ }^{-1}$ patient (DF250) primary BMSCs were treated with increasing doses of taxol for $10 \mathrm{~h}$, and the mitotic index was quantitated as in E. BMSCs from 2 different SDS patients (DF250 and DF259) yielded similar results. $\# P=0.11$; $\# P=0.09$. (G) $S B D S^{-/-}$cells are resistant to micronuclei formation following taxol treatment. Normal control or SDS patient (DF250) primary BMSCs were treated with increasing concentrations of taxol for $10 \mathrm{~h}$. Cells were stained with DAPI, and the percentage of cells with micronuclei was quantitated for at least 100 cells per experiment for 3 independent experiments. Samples were quantitated in a blinded fashion. Similar results were obtained with BMSCs from at least 2 different SDS patients. ${ }^{*} P<0.05$.

can escape from mitotic arrest to re-enter the $\mathrm{G}_{1}$ phase of the cell cycle without completing cytokinesis, through a process called "mitotic slippage" (reviewed in refs. 14, 15). Such cells would har- bor twice the normal number of chromosomes and centrosomes, thus promoting aberrations of subsequent mitoses such as those observed here. Although such polyploid cells may be eliminated 
through mechanisms such as apoptosis, such cells have been shown to propagate under certain conditions such as the loss of p53, which would impair both checkpoint activation and apoptosis (16). Interestingly, tetraploidy is also associated with an increased incidence of chromosomal breaks and translocations through mechanisms that are currently unclear (15). Elucidation of the subsequent events contributing to the development of multipolar spindles and centrosomal amplification following SBDS loss will be an important direction for future studies. Because patients with SDS do not exhibit constitutional aneuploidy, the relatively high incidence of mitotic abnormalities observed in vitro may reflect culture conditions that might allow the amplification of mitotically abnormal cells that would have been largely eliminated in vivo.

The mitotic defect of SBDS cells might lead to several downstream effects. In cells containing functional apoptotic mechanisms, we find that loss of SBDS results in apoptosis. Indeed, the bone marrow of SDS patients exhibit increased apoptosis (17) and elevated p53 expression (18). Thus, the spindle defect of SBDS cells may explain, at least in part, the bone marrow failure in SDS patients. Alternatively, in cells with compromised apoptotic or checkpoint pathways, loss of SBDS might enable more extensive genetic alterations that would promote tumorigenesis. Consistent with this idea, we find that SBDS depletion in cells lacking functional p53 results in polyploidy and aneuploidy. Similar divergent effects dependent upon the cellular or genetic context have been proposed for telomerase defects, which may either limit cell proliferation or result in genomic instability $(19,20)$. Chromosome segregation errors can lead to cytokinesis failure and tetraploidy that in turn can facilitate tumorigenesis and additional chromosomal aberrations $(16,21,22)$. Prior passage through an unstable tetraploid intermediate could also explain the extra centrosomes that we observed in $S B D S^{-/}$cells, although at this point, a role for SBDS in centrosome duplication cannot be entirely excluded. Such events may contribute to the complex chromosomal aberrations typically seen in leukemia cells from SDS patients, and studies of SBDS function in hematopoietic systems to pursue this hypothesis are ongoing.

Although SDS may affect many organ systems, the reason why SBDS mutations particularly affect the bone marrow and the pancreas remains unclear. Indeed, the tissue-specific clinical phenotypes and tissue-specific malignant manifestations exhibited following disruption of fundamental cellular processes pose a puzzling question for many of the inherited marrow failure syndromes. It is possible that some tissues are particularly dependent on the SBDS pathway to maintain spindle integrity, perhaps due to the selective deficiency of additional spindle-stabilizing factors. Another possibility is that perhaps levels of functional SBDS protein are limiting in specific tissues during mitosis. It is also possible that certain tissues are particularly susceptible to endogenous or exogenous mitotic stressors. Additional functions of the SBDS protein might also contribute to the clinical phenotype.

Current evidence indicates that SBDS is likely a multifunctional protein. Based on inferences from orthologs, SBDS has previously been hypothesized to function in rRNA processing. (1, 23-25) Recent data link SBDS and 60 S ribosome maturation $(26,27)$. In interphase cells, SBDS shuttles in and out of the nucleolus, the major cellular site of ribosome assembly (6). It is possible that differential cellular localization might reflect different SBDS functions. Additional proteins, such as nucleophosmin (28) and Rrp14
(29), have also previously been shown to play roles in both ribosome biogenesis and mitosis. Such multiplicity of a given protein's cellular function may additively contribute to disease phenotype. Dyskeratosis congenita $(30,31)$, another bone marrow failure syndrome with leukemia predisposition, provides an illustrative example of a disease in which phenotype may be affected by the multifunctional role of a single protein, dyskerin (32). Dyskerin plays a role both in rRNA pseudouridylation $(33,34)$ and ribosomal function (35) as well as in telomerase function $(34,36,37)$. Patients with dyskerin mutations typically exhibit a more severe phenotype than patients with mutations in TERC or TERT, which affect only telomerase.

In summary, we have demonstrated that the SBDS protein has a critical role in stabilizing the mitotic spindle. Further study of the potential role of microtubule stabilizing agents in the treatment of SDS patients with bone marrow failure is warranted.

\section{Methods}

Patient samples. Blood and/or bone marrow samples were obtained following Dana Farber Cancer Institute Institutional Review Board-approved informed consent. SDS patients were diagnosed on clinical grounds with genetic testing to confirm the presence of SBDS mutations.

Cell lines and cloning. Patient-derived cell cultures were established and maintained from either peripheral blood or bone marrow aspirates as previously described (6). GM00038 or GM00637 (Corielle Cell Repositories) are wild-type skin fibroblasts transformed with SV40 T-Ag or SV40 virus, respectively. Lentivirus vectors bearing a dual-cassette construct expressing GFP under a CMV promoter followed by an IRES sequence upstream of the SBDS cDNA were used (38). SBDS knockdown was performed using 3 separate siRNA hairpins in the LentiLox3.7 system (39). The following sequences were used to target siRNAs against SBDS, AACATGCTGCCATAACTTAGATT, AAGCTTGGATGATGTTCCTGATT, and AAGGAAGATCTCATCAGTGCGTT.

Viral infection. Retroviral production and infections were performed as previously described (6). Lentiviral production was performed as previously described (38). Lentiviral infection was achieved using a MOI of 50, with $5 \mu \mathrm{g} / \mathrm{ml}$ hexadimethrine bromide (Sigma-Aldrich), in $250 \mu \mathrm{l}$ total volume for lymphoblast cells and in $5 \mathrm{ml}$ total volume for GM00038 fibroblasts. Infected cells were sorted for GFP expression by flow cytometry.

Immunoblotting. Western blot analysis was performed as previously described (6) using antibodies against SBDS (6) (1:5000), PARP (Cell Signaling and Calbiochem/EMD Biosciences Inc.) (1:500), caspase-3 (Abcam) (1:800), or anti- $\alpha$-tubulin (Sigma-Aldrich) (1:2000).

Immunofluorescence. Immunofluorescence was performed as previously described (6) using antibodies against SBDS (6) (1:400), pericentrin (Abcam) (1:800), $\alpha$-tubulin (Sigma-Aldrich) (1:800), or CREST (1:1000).

Flow cytometry. Annexin $\mathrm{V}$ binding was performed on lymphoblast cells treated for $18 \mathrm{~h}$ with either $2 \mu \mathrm{g} / \mathrm{ml}$ nocodazole (Sigma-Aldrich) or $50 \mathrm{nM}$ taxol (Sigma-Aldrich). Cells were washed in PBS, resuspended in $500 \mu \mathrm{l}$ annexin $V$ buffer (BD Biosciences - Pharmingen), and incubated with $1 \mu \mathrm{l}$ anti-annexin $\mathrm{V}$ antibody conjugated to Cy3 (BD Biosciences - Pharmingen) at room temperature for 5 minutes.

For flow cytometric analysis of DNA content, cells were fixed in ethanol and washed in PBS. Pellets were resuspended in $100 \mu \mathrm{g} / \mathrm{ml}$ propidium iodide (Sigma-Aldrich) and $300 \mathrm{mU} / \mathrm{ml} \mathrm{RNase} \mathrm{(Roche)} \mathrm{in} \mathrm{PBS} \mathrm{for} 10 \mathrm{~min}$ utes at $37^{\circ} \mathrm{C}$ and were strained through $70-\mu \mathrm{m}$ cell strainers, and DNA content was measured using a FACSCalibur flow cytometer (BD Biosciences) with FlowJo software (Tree Star).

Cell survival assays. Lymphoblasts were plated in 96-well plates (Corning) at a density of $2 \times 10^{6} \mathrm{cells} / \mathrm{ml}$ in $100 \mu \mathrm{l}$ volumes of RPMI. Serial dilutions 
of either nocodazole or taxol were added in $100 \mu \mathrm{l}$ volumes of RPMI. Viability was determined using AqueousOne Cell Titer reagent (Promega).

Microtubule binding and stability assays. For the microtubule co-sedimentation assays, wild-type cells were lysed in NETN buffer $(50 \mathrm{mM}$ Tris-base, $\mathrm{pH}$ 7.4, $150 \mathrm{mM} \mathrm{NaCl}, 5 \mathrm{mM}$ EDTA, $0.2 \mathrm{mM}$ PMSF, $1 \mu \mathrm{M}$ pepstatin, and $2 \mathrm{X}$ protease inhibitor tablet [Roche]). Recombinant his-tagged SBDS was purified using the HAT expression system (Invitrogen) followed by linear gradient cation exchange chromatography $(75-1000 \mathrm{mM} \mathrm{NaCl} ; 50 \mathrm{mM}$ HEPES, pH 7.4, Uno S column; Bio-Rad). SBDS samples were diluted into BRB80 buffer ( $80 \mathrm{mM}$ PIPES, $1 \mathrm{mM}$ EGTA, $1 \mathrm{mM} \mathrm{MgCl}$, pH 6.9) supplemented with $75 \mathrm{mM} \mathrm{NaCl}, 0.2 \mathrm{mg} / \mathrm{ml}$ casein, and $10 \mu \mathrm{M}$ taxol and then precleared by centrifugation at $150,000 \mathrm{~g}$ for $15 \mathrm{~min}$ at $4^{\circ} \mathrm{C}$. Pre-assembled, taxol-stabilized microtubules in the same buffer were added and the samples incubated for $10 \mathrm{~min}$ on the bench. Subsequently, the microtubules were pelleted at $100,000 \mathrm{~g}$ for $15 \mathrm{~min}\left(25^{\circ} \mathrm{C}\right)$ through a $40 \%$ glycerol cushion in the same buffer (without casein). SBDS protein in equivalent volumes of supernatant and pellet was detected by western blotting using an Alpha Innotech Imager with CCD camera and quantified with Slidebook image analysis software (Olympus).

To determine microtubule stabilizing activity, recombinant his-tagged SBDS (purified by the HAT system and dialyzed into PBS) was added at concentrations of 50,100 , and $200 \mu \mathrm{g} / \mathrm{ml}$ and the microtubule stability assay was performed per the manufacturer's instructions (Cytoskeleton).

Statistics. Unless otherwise noted, data are presented as the mean \pm SEM. $P$ values were calculated using the 2 -tailed Student's $t$ test.

\section{Acknowledgments}

We are grateful to David G. Nathan for helpful discussions. We thank R. Himes and J. Huff for the gift of tubulin protein. We thank Alex Balazs for technical assistance and advice. We thank all patients, their families, and referring physicians. This work was supported by grants from the NIH to A. Shimamura (7R01 HL079582-06) and D. Pellman, an ACS postdoctoral fellowship to M.L. Gupta Jr., and support from the Gracie Fund and the V Foundation to A. Shimamura.

Received for publication August 29, 2007, and accepted in revised form January 30, 2008.

Address correspondence to: Akiko Shimamura, Department of Pediatric Hematology/Oncology, University of Washington, 815 Mercer St., Room 356, Seattle, Washington 98109, USA. Phone: (206) 6855282; Fax: (206) 616-4082; E-mail: shima2@u.washington.edu.
1. Dror, Y. 2005. Shwachman-Diamond syndrome. Pediatr. Blood Cancer. 45:892-901.

2. Shimamura, A. 2006. Shwachman-Diamond syndrome. Semin. Hematol. 43:178-188.

3. Boocock, G.R., et al. 2003. Mutations in SBDS are associated with Shwachman-Diamond syndrome. Nat. Genet. 33:97-101.

4. Zhang, S., Shi, M., Hui, C.C., and Rommens, J.M. 2006. Loss of the mouse ortholog of the shwachman-diamond syndrome gene (sbds) results in early embryonic lethality. Mol. Cell. Biol. 26:6656-6663.

5. Woloszynek, J.R., et al. 2004. Mutations of the SBDS gene are present in most patients with ShwachmanDiamond syndrome. Blood. 104:3588-3590.

6. Austin, K.M., Leary, R.J., and Shimamura, A. 2005 The Shwachman-Diamond SBDS protein localizes to the nucleolus. Blood. 106:1253-1258.

7. Rawls, A.S., Gregory, A.D., Woloszynek, J.R., Liu, F., and Link, D.C. 2007. Lentiviral-mediated RNAi inhibition of Sbds in murine hematopoietic progenitors impairs their hematopoietic potential. Blood. 110:2414-2422.

8. Dror, Y., et al. 2002. Clonal evolution in marrows of patients with Shwachman-Diamond syndrome: a prospective 5-year follow-up study. Exp. Hematol. 30:659-669.

9. Maserati, E., et al. 2006. Shwachman syndrome as mutator phenotype responsible for myeloid dysplasia/neoplasia through karyotype instability and chromosomes 7 and 20 anomalies. Genes Chromosomes Cancer. 45:375-382.

10. Cheeseman, I.M., Chappie, J.S., Wilson-Kubalek, E.M., and Desai, A. 2006. The conserved KMN network constitutes the core microtubule-binding site of the kinetochore. Cell. 127:983-997.

11. Rieder, C.L., and Maiato, H. 2004. Stuck in division or passing through: what happens when cells cannot satisfy the spindle assembly checkpoint. Dev. Cell. 7:637-651.

12. Rowinsky, E.K., and Donehower, R.C. 1995. Paclitaxel (taxol). N. Engl. J. Med. 332:1004-1014.

13. Hanks, S., et al. 2004. Constitutional aneuploidy and cancer predisposition caused by biallelic mutations in BUB1B. Nat. Genet. 36:1159-1161.
14. Ganem, N.J., and Pellman, D. 2007. Limiting the proliferation of polyploid cells. Cell. 131:437-440.

15. Ganem, N.J., Storchova, Z., and Pellman, D. 2007. Tetraploidy, aneuploidy and cancer. Curr. Opin. Genet. Dev. 17:157-162

16. Fujiwara, T., et al. 2005. Cytokinesis failure generating tetraploids promotes tumorigenesis in p53null cells. Nature. 437:1043-1047.

17. Dror, Y., and Freedman, M.H. 2001. ShwachmanDiamond syndrome marrow cells show abnormally increased apoptosis mediated through the Fas pathway. Blood. 97:3011-3016.

18. Elghetany, M.T., and Alter, B.P. 2002. p53 protein overexpression in bone marrow biopsies of patients with Shwachman-Diamond syndrome has a prevalence similar to that of patients with refractory anemia. Arch. Pathol. Lab. Med. 126:452-455.

19. Hackett, J.A., and Greider, C.W. 2002. Balancing instability: dual roles for telomerase and telomere dysfunction in tumorigenesis. Oncogene. 21:619-626.

20. Sharpless, N.E., and DePinho, R.A. 2004. Telomeres, stem cells, senescence, and cancer. J. Clin. Invest. 113:160-168.

21. Shi, Q., and King, R.W. 2005. Chromosome nondisjunction yields tetraploid rather than aneuploid cells in human cell lines. Nature. 437:1038-1042.

22. Storchova, Z., and Pellman, D. 2004. From polyploidy to aneuploidy, genome instability and cancer. Nat. Rev. Mol. Cell Biol. 5:45-54.

23. Peng, W.T., et al. 2003. A panoramic view of yeast noncoding RNA processing. Cell. 113:919-933.

24. Shammas, C., et al. 2005. Structural and mutational analysis of the SBDS protein family: insight into the leukemia-associated Shwachman-Diamond Syndrome. J. Biol. Chem. 280:19221-19229.

25. Savchenko, A., et al. 2005. The Shwachman-Bodian-diamond syndromeprotein family is involved in RNA metabolism. J. Biol. Chem. 280:19213-19220.

26. Menne, T.F., et al. 2007. The Shwachman-BodianDiamond syndrome protein mediates translational activation of ribosomes in yeast. Nat. Genet. 39:486-495.

27. Ganapathi, K.A., et al. 2007. The human Shwachman-Diamond syndrome protein, SBDS, associ- ates with ribosomal RNA. Blood. 110:1458-1465.

28. Grisendi, S., Mecucci, C., Falini, B., and Pandolfi, P.P. 2006. Nucleophosmin and cancer. Nat. Rev. Cancer. 6:493-505.

29. Oeffinger, M., Fatica, A., Rout, M.P., and Tollervey, D. 2007. Yeast Rrp14p is required for ribosomal subunit synthesis and for correct positioning of the mitotic spindle during mitosis. Nucleic Acids Res. 35:1354-1366.

30. Dokal, I. 2000. Dyskeratosis congenita in all its forms. Br. J. Haematol. 110:768-779.

31. Vulliamy, T., and Dokal, I. 2006. Dyskeratosis congenita. Semin. Hematol. 43:157-166.

32. Heiss, N.S., et al. 1998. X-linked dyskeratosis congenita is caused by mutations in a highly conserved gene with putative nucleolar functions. Nat. Genet. 19:32-38.

33. Ruggero, D., et al. 2003. Dyskeratosis congenita and cancer in mice deficient in ribosomal RNA modification. Science. 299:259-262.

34. Mochizuki, Y., He, J., Kulkarni, S., Bessler, M., and Mason, P.J. 2004. Mouse dyskerin mutations affect accumulation of telomerase RNA and small nucleolar RNA, telomerase activity, and ribosomal RNA processing. Proc. Natl. Acad. Sci. U. S. A. 101:10756-10761.

35. Yoon, A., et al. 2006. Impaired control of IRESmediated translation in X-linked dyskeratosis congenita. Science. 312:902-906.

36. Mitchell, J.R., Wood, E., and Collins, K. 1999. A telomerase component is defective in the human disease dyskeratosis congenita. Nature. 402:551-555.

37. Vulliamy, T., et al. 2001. The RNA component of telomerase is mutated in autosomal dominant dyskeratosis congenita. Nature. 413:432-435.

38. Mostoslavsky, G., Fabian, A.J., Rooney, S., Alt, F.W., and Mulligan, R.C. 2006. Complete correction of murine Artemis immunodeficiency by lentiviral vector-mediated gene transfer. Proc. Natl. Acad. Sci. U.S. A. 103:16406-16411.

39. Rubinson, D.A., et al. 2003. A lentivirus-based system to functionally silence genes in primary mammalian cells, stem cells and transgenic mice by RNA interference. Nat. Genet. 33:401-406. 